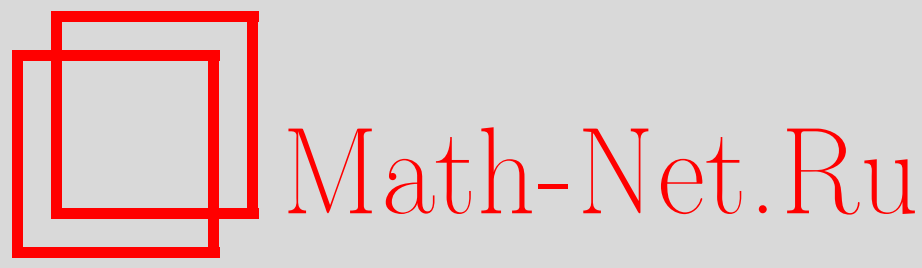

А. Д. Суханов, K квантовому обобщению равновесной статистической термодинамики. Эффективные макропараметры, ТМФ, 2008, том 154, номер 1, 183-196

DOI: https://doi.org/10.4213/tmf6159

Использование Общероссийского математического портала Math-Net.Ru подразумевает, что вы прочитали и согласны с пользовательским соглашением http://www.mathnet.ru/rus/agreement

Параметры загрузки:

IP : 54.210 .77 .194

26 апреля 2023 г., 11:09:16

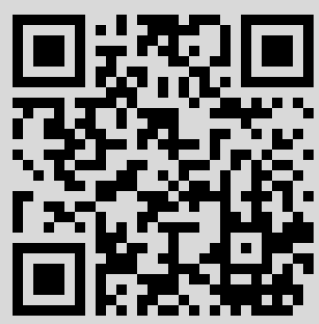




\title{
К КВАНТОВОМУ ОБОБШЕНИЮ РАВНОВЕСНОЙ СТАТИСТИЧЕСКОЙ ТЕРМОДИНАМИКИ. ЭФФЕКТИВНЫЕ МАКРОПАРАМЕТРЫ
}

\begin{abstract}
Предложено обобщение статистической термодинамики, в котором квантовые эффекты учитываются на макроуровне без явного использования операторного формализма при сохранении традиционных соотношений между макропараметрами. В обобщенной модели термостата тепловое равновесие характеризуется эффективной температурой, имеющей ограничение снизу. Введены фундаментальные макропараметры теории - эффективная энтропия и эффективное действие. Эффективная энтропия при низких температурах отлична от нуля, что позволяет придать третьему началу термодинамики форму, постулированную Нернстом. Эффективное действие при любой температуре совпадает с произведением стандартных отклонений координаты и импульса в соотношении неопределенностей Гейзенберга и потому ограничено снизу. Установлено, что в пределе низких температур отношение эффективного действия к эффективной энтропии определяется константой целостного стохастического воздействия, зависящей от постоянных Планка и Больцмана. Показано, что те же результаты могут быть получены в рамках модифицированной версии термополевой динамики, в которой квантовый осциллятор описывается комплексной макроскопической волновой функцией, зависящей от температуры. Исследовано различие в поведении отношения действия к энтропии в пределе низких температур в предлагаемой теории и в квантовой равновесной статистической механике, что может быть проверено экспериментально.
\end{abstract}

Ключевые слова: квантовый термостат, эффективная температура, эффективная энтропия, эффективное действие, константа стохастического воздействия.

\section{1. ПРОБЛЕМА ОБОБЩЕНИЯ РАВНОВЕСНОЙ ТЕРМОДИНАМИКИ}

В последние годы наблюдается возросший интерес к использованию равновесной термодинамики как самостоятельной макротеории. Эту тенденцию можно объяснить по крайней мере двумя обстоятельствами. С фундаментальной точки зрения термодинамика дает универсальное макроописание природы, в котором можно не использовать конкретные микромодели объектов. В современных условиях роль универсального безмодельного описания резко возрастает. С прагматической точки

*Объединенный институт ядерных исследований, г. Дубна, Московская обл., Россия. E-mail: ogol@oldi.ru 
зрения, в свою очередь, наметилась потребность в применении термодинамики как к относительно малым объектам (наночастицы, ядерные спины и т.п.), находящимся в тепловом равновесии при низких температурах, так и в физике высоких энергий, включая кварк-глюонную плазму, и в моделях ранней Вселенной.

Как известно, равновесная термодинамика базируется на четырех началах. Среди них исходным является нулевое начало, фиксирующее фундаментальное понятие теплового равновесия объекта с его макроокружением, называемым термостатом. В классической термодинамике, в которой все макропараметры определены точно, нулевое начало имеет вид жесткого условия, определяющего понятие температуры:

$$
T \equiv T_{0}
$$

где $T$ - температура объекта, а $T_{0}$ - температура термостата.

В статистической термодинамике [1]-[5], обобщение которой является предметом данной работы, все макропараметры рассматриваются как случайные величины, испытывающие флуктуации около своих средних значений. При этом предполагается, что понятие теплового равновесия сохраняется. Однако его содержание обобщается, ибо теперь допускается, что температура объекта благодаря тепловому стохастическому воздействию термостата, характеризуемому постоянной Больцмана $k_{\mathrm{B}}$, также способна испытывать флуктуации. В результате нулевое начало принимает вид мягкого условия

$$
T=T_{0} \pm \delta T,
$$

так что средняя температура объекта $\langle T\rangle$ совпадает с $T_{0}$, а $\delta T=\sqrt{\left\langle(\Delta T)^{2}\right\rangle}$ имеет смысл среднеквадратичного отклонения от $\langle T\rangle$.

Для сохранения термодинамического характера описания одновременно предполагается, что величины флуктуаций любых макропараметров $A_{i}$ ограничены условием $\left(\delta A_{i}\right)^{2} /\left\langle A_{i}\right\rangle^{2} \leqslant 1$. Это означает, что на величину $\delta T$ в формуле (2) наложено следующее требование:

$$
\frac{(\delta T)^{2}}{T_{0}^{2}} \leqslant 1 .
$$

Иными словами, нулевое начало существующей ныне неквантовой версии равновесной статистической термодинамики - это не одно только условие (2), а совокупность условий (2) и (3).

Нетрудно, однако, видеть, что данная форма нулевого начала при определенных условиях приходит в противоречие с опытом, из которого известно, что с уменьшением числа частиц, объема и температуры условие (3) может быть нарушено [6], [7]. Как заметил Д. Н. Зубарев [8], реальная область значений температур, в которой следует считаться с возможностью нарушения условия (3), оказывается довольно широкой. Она может начинаться со значений температур вырождения идеальных газов или даже температуры Дебая в кристаллах.

Кроме того, традиционная статистическая термодинамика абсолютно не учитывает квантовое стохастическое воздействие, характеризуемое постоянной Планка $\hbar$ (см. гл. 12 в книге [9]). В то же время из квантовой динамики известно, что в отсутствие тепловых эффектов характеристики объекта могут испытывать чисто квантовые флуктуации. В общем случае на опыте наблюдаются одновременно и квантовый, и тепловой типы стохастических воздействий макроокружения, определяющие как сами макропараметры, так и их флуктуации. В связи с этим возникает 
необходимость создания теории, которая позволила бы в каком-то смысле соединить подходы собственно квантовой динамики и традиционной статистической термодинамики.

Сегодня довольно широко распространено убеждение, что роль такой теории давно и весьма эффективно выполняет квантовая равновесная статистическая механика (KPCM) [9]-[11]. Однако для решения ряда новых задач эта теоретическая модель, по-видимому, оказывается непригодной. На наш взгляд, это обусловлено следующими существенными факторами. Во-первых, КРСМ не является последовательной квантовой теорией, поскольку, как будет подробнее аргументировано ниже, она играет роль квазиклассического приближения, в котором фактически не учитывается наличие ненулевой энергии основного состояния. Во-вторых, она не является последовательной статистической теорией, ибо в ней флуктуации интенсивных макропараметров (прежде всего, температуры) отсутствуют изначально, а флуктуации экстенсивных макропараметров обращаются в нуль в термодинамическом пределе.

В связи с этим для квантового обобщения статистической термодинамики в принципе возможны два пути. Один из них - развитие квантовой неравновесной статистической механики как микротеории [12] с последовательным включением в нее тепловых флуктуаций макропараметров, обусловленных флуктуациями температуры. С нашей точки зрения, предпочтительнее избрать другой путь, позволяющий сохранить язык равновесной статистической термодинамики при последовательном учете квантовых эффектов непосредственно на макроуровне. Главное, что следует сделать, - оставаясь в рамках взглядов Гиббса и Эйнштейна на тепловое равновесие, фиксируемых нулевым началом в форме (2), (3), - это обеспечить в рамках макротеории использование квантовых идей уже на уровне таких понятий как модель термостата.

В данной работе мы приступаем к изложению статистической макротеории, представляющей собой квантовое обобщение равновесной статистической термодинамики $(\mathrm{KOPCT})^{1)}$. При ее построении мы будем опираться на основной постулат, сводящийся к совокупности следующих утверждений:

А. Стохастические воздействия квантового и теплового типов сосредоточены в термостате, которому сопоставляется квантовая модель.

Б. Состояние теплового равновесия между объектом и термостатом описывается эффективной температурой.

В. Физические характеристики объектов любой сложности при любой температуре характеризуются эффективными макропараметрами, которым сопоставляются случайные с-числовые величины.

Г. Основные термодинамические соотношения при сохранении их стандартного вида, включая нулевое начало в форме (2), (3), формулируются для соответствующих эффективных макропараметров.

\section{2. ЭФФЕКТИВНАЯ ТЕМПЕРАТУРА}

Приступая к квантовому обобщению равновесной статистической термодинамики как макротеории, отметим, что при изменении формы нулевого начала от (1)

\footnotetext{
1) Заметим, что встречающиеся в литературе термины типа "квантовая термодинамика" или “термодинамика простых квантовых систем" на самом деле относятся к КРСМ и потому не имеют никакого отношения к рассматриваемому здесь КОРСТ.
} 
к (2), (3) допустимость флуктуаций температуры объекта была уже учтена. Поэтому единственная возможность, которая, по-видимому, еще осталась, - это модифицировать модель самого термостата, являющегося источником стохастических воздействий, органично включив в их число и воздействие квантового типа.

Поскольку явная попытка модификации модели термостата здесь предпринимается впервые, полезно сначала проанализировать, какая модель термостата по умолчанию принимается в неквантовой версии статистической термодинамики. В ее основе, как это следует [5] из главы IX книги Гиббса [1], лежит каноническое распределение в пространстве макропараметров

$$
d w(\mathcal{E})=e^{(F-\mathcal{E}) / \Theta} d \mathcal{E} .
$$

В нем энергия объекта $\mathcal{E}=\mathcal{E}(V, T)$ является случайной величиной, флуктуации которой (при $V=$ const) определяются флуктуациями температуры объекта в соответствии с нулевым началом (2), (3), F- свободная энергия, определяемая условием нормировки, а в качестве модуля распределения $\Theta$ выбирается энергия, характерная для определенной модели термостата ${ }^{2)}$ :

$$
\Theta=k_{\mathrm{B}} T_{0} .
$$

Согласно идеям Боголюбова [10] и Фейнмана [11] термостат до сих пор принято моделировать бесконечной совокупностью нормальных мод, каждую из которых можно трактовать как возбуждение цепочки слабо связанных осцилляторов. Как следует из опыта, величина $k_{\mathrm{B}} T_{0}$ в относительно узком диапазоне частот и температур имеет смысл средней энергии классической нормальной моды $\left\langle\varepsilon_{\mathrm{cl}}\right\rangle$. Поэтому из формул (4) и (5) можно заключить, что в неквантовой версии статистической термодинамики используется модель термостата, которую естественно назвать классической. С современной точки зрения классическая модель термостата, на которой, подчеркнем, основана и КРСМ, в ряде случаев не позволяет интерпретировать данные эксперимента.

Ниже предлагается альтернативный способ одновременного учета стохастических воздействий квантового и теплового типов. В соответствии с основным постулатом КОРСТ перейдем от классической модели термостата к более общей квантовой модели. Это позволит все эффекты, связанные с обоими типами стохастических воздействий макроокружения на объект, отнести на счет термостата, а в остальном сохранить термодинамический язык описания теплового равновесия, т.е. обойтись в нем без явного использования операторного формализма.

C этой целью мы предлагаем выбрать в качестве модели термостата совокупность бесконечного числа квантовых нормальных мод, средняя энергия каждой из которых во всем диапазоне частот и температур в соответствии с экспериментом равна

$$
\left\langle\varepsilon_{\mathrm{qu}}\right\rangle=\frac{\hbar \omega}{2} \operatorname{cth} \frac{\hbar \omega}{2 k_{\mathrm{B}} T_{0}}=\frac{\hbar \omega}{2}+\hbar \omega\left(e^{\hbar \omega /\left(k_{\mathrm{B}} T_{0}\right)}-1\right)^{-1} .
$$

Это означает, что в квантовой модели термостата выражение для модуля распределения $\Theta$ мы определяем более общим условием $\Theta=\left\langle\varepsilon_{\mathrm{qu}}\right\rangle$ вместо условия $\Theta=\left\langle\varepsilon_{\mathrm{cl}}\right\rangle$, характерного для классической модели.

2) Следует подчеркнуть, что распределение (4) лишь внешне совпадает с каноническим распределением из классической равновесной статистической механики, в котором энергия $\varepsilon=\varepsilon(p, q)$ также является случайной величиной, но ее флуктуации определяются флуктуациями микропараметров $p$ и $q$ при значении температуры объекта, фиксированном условием (1). 
Далее в соответствии с основным постулатом КОРСТ величине $\Theta$ предлагается придать вид [13]

$$
\Theta \equiv k_{\mathrm{B}} T_{0}^{*}
$$

Характерно, что введенная здесь величина

$$
T_{0}^{*} \equiv \frac{\left\langle\varepsilon_{\mathrm{qu}}\right\rangle}{k_{\mathrm{B}}}=\frac{\hbar \omega}{2 k_{\mathrm{B}}} \operatorname{cth} \frac{\hbar \omega}{2 k_{\mathrm{B}} T_{0}}
$$

зависит от обеих фундаментальных постоянных $\hbar$ и $k_{\mathrm{B}}$. Она имеет смысл эффективной температуры квантового термостата, фиксирующей условие теплового равновесия при равноправном учете обоих типов стохастических воздействий ${ }^{3)}$.

Теперь нулевое начало можно было бы сформулировать по аналогии с формулами (2), (3) как условие взаимосвязи эффективных температур объекта $T^{*}$ и квантового термостата $T_{0}^{*}$. Однако в данной работе мы ограничимся рассмотрением круга вопросов, при исследовании которых флуктуации эффективной температуры объекта можно не учитывать, так что ниже во всех формулах мы положим $T^{*} \equiv T_{0}^{*}$. Квантовое обобщение теории флуктуаций макропараметров будет предметом отдельной публикации.

Обратим внимание на то, что эффективная температура объекта $T^{*}$ является функцией двух его характеристик $\omega$ и $T$. При этом в качестве квантового термостата с температурой $T$ по шкале Кельвина фактически выступает равновесное тепловое излучение со сплошным спектром. В этих условиях при достижении состояния теплового равновесия не только $T=T_{0}$, но и $\omega=\omega_{0}$, т.е. объект как бы осуществляет резонансный выбор одной из мод термостата, совпадающей по частоте с его характерной частотой $\omega$. Соответствующую частоту необходимо выбирать из опыта или каких-либо интуитивных соображений. В связи с этим можно допустить, что КОРСТ применимо, по крайней мере, к обширному классу объектов, для которых независимым степеням свободы можно сопоставить периодические или условно периодические движения.

Следует заметить, что частота $\omega$ и температура $T$ (и тем самым эффективная температура $T^{*}$ ) являются интенсивными величинами, что подчеркивает их концептуальную близость друг другу, но делает их качественно отличными от экстенсивных величин (например, энергии), для которых характерна аддитивность. Они являются целостными характеристиками состояния системы "объект плюс макроокружение", обладающими свойством транзитивности. Существенно, что характеристики $\omega, T$ и $T^{*}$, являясь $c$-числовыми величинами, исходно не относятся к числу наблюдаемых, которым в квантовой теории сопоставляются операторы.

Физический смысл эффективной температуры $T^{*}$ можно пояснить, рассматривая ее поведение в предельных ситуациях. Так, если температура по шкале Кельвина $T \rightarrow 0$, эффективная температура $T^{*}$ достигает отличного от нуля значения ${ }^{4}$

$$
T^{*} \rightarrow T_{\min }^{*} \equiv \frac{\hbar \omega}{2 k_{\mathrm{B}}},
$$

\footnotetext{
3) Косвенным образом подобная идея была высказана Эйнштейном еще в 1914 г. в статье, посвященной термодинамическим проблемам, но имевшей тем не менее характерное название "K квантовой теории" [14].

4) Необходимо отметить, что выражения, которым можно было бы придать вид (8) и (9), впервые фактически появились в работах Блоха [15] и Вигнера [16], хотя и в ином контексте. В последующие годы они использовались и другими авторами для решения ряда частных задач (см., например, [17]-[19]).
} 
имеющего смысл минимальной эффективной температуры для объекта с характерной частотой $\omega$. Тем самым даже при $T \rightarrow 0$ стохастическое воздействие не сводится к чисто квантовому.

В свою очередь, в пределе высоких температур $T$ эффективная температура принимает вид

$$
T^{*} \equiv T_{\min }^{*} \operatorname{cth} \frac{T_{\min }^{*}}{T} \rightarrow T\left[1+\frac{1}{3}\left(\frac{T_{\min }^{*}}{T}\right)^{2}+\cdots\right] .
$$

Тем самым даже при $T \gg T_{\min }^{*}$ стохастическое воздействие не сводится к чисто тепловому. Разумеется, сами понятия низких и высоких температур для каждого объекта существенно зависят от отношения $T_{\min }^{*} / T$.

\section{3. ЭФФЕКТИВНАЯ ЭНТРОПИЯ}

Для вычисления эффективных макропараметров через соответствующую функцию распределения необходимо обобщить каноническое распределение (4), введенное Гиббсом в неквантовой версии статистической термодинамики [1]. Это обобщение в соответствии с основным постулатом КОРСТ сводится к замене выражения (5) для модуля распределения $\Theta$ выражением (7), т.е. к замене $T=T_{0}$ на $T^{*}=T_{0}^{*}$. В результате искомое распределение принимает вид

$$
d w(\mathcal{E})=\rho(\mathcal{E}) d \mathcal{E}=\frac{1}{k_{\mathrm{B}} T^{*}} e^{-\mathcal{E} /\left(k_{\mathrm{B}} T^{*}\right)} d \mathcal{E},
$$

где $\mathcal{E}$ - случайное значение энергии независимой степени свободы объекта, которой сопоставляется модель осциллятора с частотой $\omega$.

Основываясь на распределении (11), можно вычислить внутреннюю энергию такого объекта как макропараметр:

$$
\mathcal{E}^{*}=\int \mathcal{E} \rho(\mathcal{E}) d \mathcal{E}=k_{\mathrm{B}} T^{*}
$$

Поскольку $\mathcal{E}^{*}$ совпадает с $\left\langle\varepsilon_{\text {qu }}\right\rangle$ вида $(6)$, это означает, что в качестве модели объекта выбирается квантовый осциллятор в термостате.

Для вычисления эффективной энтропии $S^{*}$ такого объекта формуле (11) удобно придать вид, при котором плотность распределения $\tilde{\rho}(\mathcal{E})=\rho(\mathcal{E}) \hbar \omega / 2$ окажется безразмерной:

$$
d w(\mathcal{E})=\tilde{\rho}(\mathcal{E})\left(\frac{\hbar \omega}{2}\right)^{-1} d \mathcal{E}=e^{\left(F^{*}-\mathcal{E}\right) /\left(k_{\mathrm{B}} T^{*}\right)}\left(\frac{\hbar \omega}{2}\right)^{-1} d \mathcal{E}
$$

где эффективная свободная энергия

$$
F^{*}=-k_{\mathrm{B}} T^{*} \ln \left(\frac{T^{*}}{T_{\min }^{*}}\right) .
$$

Тогда получим

$$
\begin{aligned}
S^{*} & =-k_{\mathrm{B}} \int \tilde{\rho}(\mathcal{E}) \ln \tilde{\rho}(\mathcal{E})\left(\frac{\hbar \omega}{2}\right)^{-1} d \mathcal{E}=k_{\mathrm{B}}\left[1+\ln \left(\operatorname{cth} \frac{\hbar \omega}{2 k_{\mathrm{B}} T}\right)\right]= \\
& =k_{\mathrm{B}}\left[1+\ln \left(\operatorname{cth} \frac{T_{\text {min }}^{*}}{T}\right)\right] .
\end{aligned}
$$


Из формулы (15) следует, что в пределе высоких температур $T \gg T_{\min }^{*}$ эффективная энтропия

$$
S^{*} \rightarrow k_{\mathrm{B}} \ln T+\mathrm{const},
$$

что совпадает с выражением для энтропии осциллятора в термостате из классической термодинамики.

В то же время в пределе низких температур $T \ll T_{\min }^{*}$ эффективная энтропия определяется универсальной постоянной $k_{\mathrm{B}}$ :

$$
S^{*} \rightarrow S_{\min }^{*}=k_{\mathrm{B}}
$$

Тем самым в КОРСТ поведение эффективной энтропии степени свободы объекта, для которой характерно периодическое движение, соответствует исходной формулировке теоремы Нернста, в которой минимальное значение энтропии отлично от нуля. Более того, область значений температур $T$, в которой $S^{*} \approx S_{\min }^{*}$, может оказаться весьма значительной в зависимости от величины отношения $T_{\min }^{*} / T$.

Очевидно, что модель квантового термостата позволяет в рамках КОРСТ объединить воздействия квантового и теплового типов, традиционно считающиеся специфическими лишь для микро- и макротеорий соответственно, в целостное стохастическое воздействие. Однако такой подход позволяет не ограничиться только обобщением традиционных макропараметров типа температуры или энтропии. Появляется возможность придать макроскопический смысл понятию действия, существенно связанного на микроуровне с квантовым типом стохастического воздействия.

\section{4. ЭФФЕКТИВНОЕ ДЕЙСТВИЕ И КОНСТАНТА ЦЕЛОСТНОГО СТОХАСТИЧЕСКОГО ВОЗДЕЙСТВИЯ}

Проблема введения понятия действия в термодинамику и установления взаимосвязи двух широко известных, но используемых обычно в разных разделах физики величин - энтропии и действия - привлекала внимание самых выдающихся физиков, включая Больцмана [20], Богуславского [21], де Бройля [22] и многих других. Однако полученные до сих пор результаты в основном относились к классической термодинамике, а квантовые эффекты учитывались лишь в квазиклассическом приближении [21]. Наша цель - распространить их на КОРСТ.

Для этого в качестве исходной модели объекта, совершающего периодическое движение, выберем гармонический осциллятор. Если в рамках классической механики при его анализе перейти от переменных $p, q$ к переменным действие-угол, то действие $j$, имеющее смысл обобщенного импульса, может быть выражено через энергию осциллятора $\varepsilon$ формулой

$$
j=\frac{\varepsilon}{\omega} .
$$

При переходе к термодинамике взаимосвязь (18) между действием и энергией желательно сохранить, но при этом перейти от микропараметров $j$ и $\varepsilon$ к соответствующим макропараметрам - средним величинам $\langle j\rangle$ и $\langle\varepsilon\rangle$, конкретные выражения для которых зависят от выбора модели термостата, используемой при усреднении.

Вполне естественно, что в рамках классической термодинамики Больцман положил

$$
\mathcal{J} \equiv\langle j\rangle=\frac{\left\langle\varepsilon_{\mathrm{cl}}\right\rangle}{\omega}=\frac{k_{\mathrm{B}} T}{\omega} .
$$


Следуя той же идее, мы определим эффективное действие $\mathcal{J}^{*}$ в рамках КОРСТ формулой

$$
\mathcal{J}^{*}=\langle j\rangle=\frac{\left\langle\varepsilon_{\mathrm{qu}}\right\rangle}{\omega}=\frac{\mathcal{E}^{*}}{\omega}=\frac{k_{\mathrm{B}} T^{*}}{\omega} .
$$

Это означает, что в КОРСТ мы исходим из того, что для всех объектов, к которым применима модель квантового осциллятора в термостате, эффективное действие имеет вид

$$
\mathcal{J}^{*}=\frac{\hbar}{2} \operatorname{cth} \frac{\hbar \omega}{2 k_{\mathrm{B}} T}=\mathcal{J}_{\min }^{*} \operatorname{cth} \frac{T_{\min }^{*}}{T} .
$$

Здесь

$$
\mathcal{J}_{\min }^{*}=\frac{\hbar}{2}
$$

- предельное значение эффективного действия при $T \ll T_{\min }^{*}$. Разумеется, в пределе $T \gg T_{\min }^{*}$ эффективное действие $\mathcal{J}^{*} \rightarrow \mathcal{J}$, т.е. совпадает с полученным в рамках классической термодинамики выражением (19).

Чтобы установить взаимосвязь действия и энтропии, Больцман предположил [20], что изотермическим процессам в термодинамике соответствуют изоциклические движения осциллятора в механике, при которых $\omega=$ const. В этом случае изменение энергии осциллятора может происходить за счет внешних воздействий, которые можно трактовать как работу диссипативных сил $\delta A_{\text {dis }}$, эквивалентную теплоте $\delta Q$.

Обобщая эту идею, мы предполагаем, что в КОРСТ всякое стохастическое воздействие (как квантовое, так и тепловое) можно трактовать как эффективную работу диссипативных сил $\delta A_{\mathrm{dis}}^{*}$, эквивалентную эффективной теплоте $\delta Q^{*}$. Это означает, что одно и то же изменение эффективной энергии $d \mathcal{E}^{*}$ макрообъекта, которому можно сопоставить модель квантового осциллятора в термостате, при изотермических процессах можно представить в двух формах

$$
d \mathcal{E}^{*}=\delta A_{\mathrm{dis}}^{*}=\omega d \mathcal{J}^{*} \quad \text { или } \quad d \mathcal{E}^{*}=\delta Q^{*}=T^{*} d S^{*} .
$$

Далее, следуя Больцману, в качестве меры передачи энергии от термостата к объекту при таких процессах выберем отношение $d \mathcal{E}^{*} / \mathcal{E}^{*}$, где числитель и знаменатель с помощью формул (23) и (20) можно выразить либо через эффективное действие $\mathcal{J}^{*}$, либо через эффективную энтропию $S^{*}$ и эффективную температуру $T^{*}$. Приравнивая друг к другу полученные выражения для отношения $d \mathcal{E}^{*} / \mathcal{E}^{*}$, приходим к дифференциальному уравнению

$$
\frac{d \mathcal{E}^{*}}{\mathcal{E}^{*}}=\frac{\omega d \mathcal{J}^{*}}{\omega \mathcal{J}^{*}}=\frac{T^{*} d S^{*}}{k_{\mathrm{B}} T^{*}}
$$

связывающему эффективную энтропию и эффективное действие. Его решение имеет вид

$$
S^{*}=k_{\mathrm{B}} \int \frac{d \mathcal{J}^{*}}{\mathcal{J}^{*}}=k_{\mathrm{B}} \ln \frac{\mathcal{J}^{*}}{\mathcal{J}_{0}}=k_{\mathrm{B}} \ln \frac{\hbar}{2 \mathcal{J}_{0}} \operatorname{cth} \frac{\hbar \omega}{2 k_{\mathrm{B}} T},
$$

где $\mathcal{J}_{0}$ - произвольная постоянная размерности действия.

Выбирая в качестве $\mathcal{J}_{0}$ величину $\hbar / 2 e$, где $e$ - основание натурального логарифма, можно добиться совпадения выражения (25) с выражением для эффективной энтропии $S^{*}$ вида (15). Учитывая, что $S_{\min }^{*}=k_{\mathrm{B}}$, имеем

$$
S^{*}=S_{\min }^{*}\left[1+\ln \frac{\mathcal{J}^{*}}{\mathcal{J}_{\min }^{*}}\right]=S_{\min }^{*}\left[1+\ln \operatorname{cth} \frac{T_{\min }^{*}}{T}\right] .
$$


Заметим, что отношение эффективного действия к эффективной энтропии согласно формулам (21) и (26) равно

$$
\frac{\mathcal{J}^{*}}{S^{*}}=\frac{\mathcal{J}_{\min }^{*}}{S_{\min }^{*}} \frac{\operatorname{cth}\left(T_{\min }^{*} / T\right)}{1+\ln \operatorname{cth}\left(T_{\min }^{*} / T\right)}=\varkappa \frac{\operatorname{cth}(\varkappa \omega / T)}{1+\ln \operatorname{cth}(\varkappa \omega / T)} .
$$

В нем

$$
\varkappa \equiv \frac{\mathcal{J}_{\min }^{*}}{S_{\min }^{*}}=\frac{\hbar}{2 k_{\mathrm{B}}}
$$

представляет собой предельное значение отношения (27) при $T \ll T^{*}$.

По нашему мнению, величина

$$
\varkappa=3.82 \cdot 10^{-12} \mathrm{Kc},
$$

представляет собой не просто обозначение одной из возможных комбинаций мировых констант $\hbar$ и $k_{\mathrm{B}}$. Она имеет и самостоятельный физический смысл. Помимо того, что через нее выражается отношение $\mathcal{J}^{*} / S^{*}$ вида $(27)$ при любой температуре, она входит в определение (8) эффективной температуры

$$
T^{*}=\varkappa \omega \operatorname{cth} \frac{\varkappa \omega}{T}
$$

а также в закон смещения для равновесного теплового излучения $T / \omega_{\max }=0.7 \varkappa$. Сказанное выше позволяет высказать гипотезу, согласно которой величина $\varkappa$ играет роль константы, характеризующей целостное стохастическое воздействие на объект. Поскольку она впервые появилась в законе смещения, ее следовало бы, на наш взгляд, назвать именем Вина.

\section{5. ЭФФЕКТИВНАЯ ЭНТРОПИЯ И ЭФФЕКТИВНОЕ ДЕЙСТВИЕ В ТЕПЛОВЫХ КОГЕРЕНТНЫХ СОСТОЯНИЯХ КВАНТОВОЙ ТЕОРИИ ПОЛЯ}

До сих пор мы развивали КОРСТ как макротеорию, позволяющую совместно и равноправно учитывать стохастические воздействия квантового и теплового типов. В рамках микротеории аналогичный подход, хотя и не совсем последовательно, развивался в равновесной версии термополевой динамики (ТПД) Умэдзавы [23], [24], существенно использующей операторный формализм. В частности, введенный в ней термополевой вакуум по эффектам воздействия на ряд характеристик объектов во многом напоминает модель квантового термостата. Поэтому представляет интерес установить, насколько могут быть согласованы результаты этих двух теорий.

С этой целью сопоставим объекту, совершающему периодическое движение, модель квантового осциллятора частоты $\omega$, взаимодействующего с термополевым вакуумом. При этом будем исходить из предложенной нами ранее версии ТПД [25], [26], отличной от исходной версии [23], [24]. В работах [25], [26] было показано, что состояние теплового равновесия квантового осциллятора при микроописании имеет смысл коррелированного (теплового) когерентного состояния, которое можно описать с помощью комплексной волновой функции, зависящей от температуры. Она имеет гауссову форму как в координатном, так и в импульсном представлении. В частности,

$$
\psi(q)=\left[2 \pi(\delta q)^{2}\right]^{-1 / 4} \exp \left\{-\frac{q^{2}}{4(\delta q)^{2}}(1-i \alpha)\right\},
$$


где $(\delta q)^{2}=k_{\mathrm{B}} T^{*} /\left(m \omega^{2}\right), \alpha=(\operatorname{sh} \varkappa \omega / T)^{-1}$.

В нашей версии ТПД соотношение неопределенностей координата-импульс в форме Шредингера для квантового осциллятора (при усреднении по волновой функции (31)) является насыщенным, т.е. принимает вид равенства [26]

$$
(\mathrm{UP})^{2} \equiv(\delta p)^{2} \cdot(\delta q)^{2}=\frac{\hbar^{2}}{4}\left[\operatorname{sh} \frac{\varkappa \omega}{T}\right]^{-2}+\frac{\hbar^{2}}{4}=\frac{\hbar^{2}}{4} \operatorname{cth}^{2} \frac{\varkappa \omega}{T},
$$

где UP обозначает целостное понятие произведение неопределенностей (Uncertainties Product) [27].

Сравнение выражений (32) и (21) показывает, что величина UP является не просто математическим объектом - произведением среднеквадратичных отклонений импульса и координаты. Она совпадает с величиной эффективного действия квантового осциллятора $\mathcal{J}^{*}$, приобретая тем самым смысл целостной характеристики объекта, чувствительной к любым стохастическим воздействиям. Это позволяет придать формуле (32) следующий вид:

$$
\left(\mathcal{J}^{*}\right)^{2}=\frac{\hbar^{2}}{4}\left[\operatorname{sh} \frac{\varkappa \omega}{T}\right]^{-2}+\left(\mathcal{J}_{\text {min }}^{*}\right)^{2} .
$$

Таким образом, выражения для эффективного действия $\mathcal{J}^{*}$ в $\mathrm{KOPCT} \mathrm{и} \mathrm{в} \mathrm{пред-}$ ложенной нами версии ТПД совпадают. Чтобы добиться аналогичного результата для эффективной энтропии $S^{*}$, мы предлагаем дать ей в рамках той же версии ТПД следующее определение:

$$
S^{*} \equiv-k_{\mathrm{B}}\{\operatorname{Sp}[\tilde{\rho}(q) \ln \tilde{\rho}(q)]+\operatorname{Sp}[\tilde{\rho}(p) \ln \tilde{\rho}(p)]\},
$$

где $\tilde{\rho}(q)$ и $\tilde{\rho}(p)$ - безразмерные плотности вероятности,

$$
\tilde{\rho}(q)=\sqrt{\frac{m \omega}{\pi \hbar}}|\psi(q)|^{2}, \quad \tilde{\rho}(p)=\sqrt{\pi \hbar m \omega}|\psi(p)|^{2} .
$$

Тогда, подставляя в (34) выражение для $\psi(q)$ вида (31) и аналогичное выражение для $\psi(p)$, получим

$$
S^{*}=k_{\mathrm{B}}\left\{\frac{1}{2}\left[1+\ln \operatorname{cth} \frac{\varkappa \omega}{T}\right]+\frac{1}{2}\left[1+\ln \operatorname{cth} \frac{\varkappa \omega}{T}\right]\right\},
$$

что совпадает с выражениями (15) или (26) для эффективной энтропии $S^{*}$ в КОРСТ.

$\mathrm{C}$ помощью выражения (35) можно рассчитать эффективную температуру $T^{*}=$ $\left(d S^{*} / d \mathcal{E}^{*}\right)^{-1}$ и другие связанные с эффективной энтропией $S^{*}$ макропараметры. Тем самым использование определения эффективной энтропии (34) обеспечивает совпадение термодинамических следствий КОРСТ и данной версии ТПД. Это позволяет впредь трактовать волновую функцию теплового когерентного состояния вида (31) как макроскопическую волновую функцию, в отличие от известных микроскопических волновых функций квантового осциллятора при отсутствии термостата.

\section{6. ЭФФЕКТИВНОЕ ДЕЙСТВИЕ И ЭФФЕКТИВНАЯ ЭНТРОПИЯ В КВАНТОВОЙ РАВНОВЕСНОЙ СТАТИСТИЧЕСКОЙ МЕХАНИКЕ}

Одним из самых важных следствий развиваемого здесь КОРСТ является отличие от нуля минимальных значений как эффективного действия $\mathcal{J}^{*}$ и эффективной 
энтропии $S^{*}$ самих по себе, так и их отношения $\mathcal{J}^{*} / S^{*}$ в диапазоне температур $T \ll T_{\min }^{*}$. Это следствие находится в резком противоречии с соответствующими результатами и КРСМ, и традиционной версии ТПД [23], [24].

Действительно, в этих двух вариантах микротеории в качестве канонического распределения используется выражение

$$
w_{n}=e^{\left(F-\varepsilon_{n}\right) /\left(k_{\mathrm{B}} T\right)},
$$

являющееся прямым обобщением аналога распределения (4) в фазовом пространстве. Для энтропии квантового осциллятора в термостате согласно фон Нейману в обеих теориях справедливо выражение

$$
S=-k_{\mathrm{B}} \sum w_{n} \ln w_{n}
$$

Подчеркнем, что вклады энергии основного состояния $\hbar \omega / 2$ в каноническое распределение (36) и в выражение для энтропии (37) исчезают вследствие условия нормировки. В результате формулы (36) и (37) по определению оказываются нечувствительными к принципиально квантовым эффектам, характеризуемым ограничением соотношения неопределенностей Гейзенберга "координата-импульс". Тем самым каноническое распределение (36) и выражение для энтропии (37) исходно являются квазиклассическими.

Как известно, в рамках квазиклассического приближения для средней энергии квантового осциллятора справедлива первоначальная формула Планка

$$
\mathcal{E}_{\text {quasi }}=\frac{\hbar \omega}{e^{\hbar \omega /\left(k_{\mathrm{B}} T\right)}-1} \equiv \mathcal{E}^{*}-\frac{\hbar \omega}{2} .
$$

Подставляя в формулу (20) вместо $\mathcal{E}^{*}$ выражение $\mathcal{E}_{\text {quasi }}$ вида $(38)$, получим действие в квазиклассическом приближении [21]:

$$
\mathcal{J}_{\text {quasi }}=\frac{\mathcal{E}_{\text {quasi }}}{\omega}=\frac{\hbar}{e^{\hbar \omega /\left(k_{\mathrm{B}} T\right)}-1} \equiv \mathcal{J}^{*}-\frac{\hbar}{2} .
$$

Квазиклассический характер выражений (38) и (39) проявляется, в частности, в том, что при $T \rightarrow 0$ обе эти величины стремятся к нулю.

Для энтропии квантового осциллятора в термостате в том же приближении в соответствии с формулой (37) справедливо известное выражение

$$
S_{\text {quasi }}=-k_{\mathrm{B}}\left\{\frac{\hbar \omega}{k_{\mathrm{B}} T}\left(1-e^{\hbar \omega /\left(k_{\mathrm{B}} T\right)}\right)^{-1}+\ln \left(1-e^{-\hbar \omega /\left(k_{\mathrm{B}} T\right)}\right)\right\},
$$

которое при $T \rightarrow 0$ также стремится к нулю.

Таким образом, значение минимального отношения действия к энтропии в КРСМ и в традиционной версии ТПД, достигаемое при $T \rightarrow 0$, определяется формулой

$$
\frac{\mathcal{J}_{\text {quasi }}}{S_{\text {quasi }}} \rightarrow \frac{\hbar e^{-\hbar \omega /\left(k_{\mathrm{B}} T\right)}}{k_{\mathrm{B}}\left(\hbar \omega / k_{\mathrm{B}} T\right) e^{-\hbar \omega /\left(k_{\mathrm{B}} T\right)}}=\frac{T}{\omega} \rightarrow 0 .
$$

Итак, мы показали, что в этих микротеориях, в отличие от КОРСТ и нашей версии ТПД, не только $\mathcal{J}_{\text {quasi }} \rightarrow 0$ и $S_{\text {quasi }} \rightarrow 0$ по отдельности, но и их отношение $\mathcal{J}_{\text {quasi }} / S_{\text {quasi }}$ также стремится к нулю. Данный результат резко отличается от предела $\mathcal{J}^{*} / S^{*} \rightarrow \varkappa \neq 0$ для соответствующих эффективных величин в КОРСТ. 
Тем самым открывается возможность экспериментального сравнения двух теорий КОРСТ и КРСМ - путем измерения предельного значения этого отношения.

Интересно отметить, что выражение для энтропии вида (34) неоднократно встречалось в литературе (см., например, обзор [28]). При этом обычно говорилось, что оно имеет смысл формальной математической величины, а "правильная" с физической точки зрения энтропия имеет вид (37).

Проведенный выше анализ показывает, что все обстоит как раз наоборот. Стандартное выражение фон Неймана (37) для энтропии, принятое в КРСM, оказывается квазиклассическим и потому непригодным к использованию во всем интервале температур. В то же время выражение (35), вытекающее из определения (34) в предложенной нами версии ТПД, и выражения (15) и (26) в КОРСТ согласованы друг с другом и позволяют независимыми способами определить эффективную энтропию $S^{*}$, адекватно учитывающую оба типа стохастических воздействий при любой температуре.

\section{7. НЕКОТОРЫЕ ПЕРСПЕКТИВЫ ПРИМЕНЕНИЯ КВАНТОВОГО ОБОБЩЕНИЯ РАВНОВЕСНОЙ СТАТИСТИЧЕСКОЙ ТЕРМОДИНАМИКИ}

Выше были изложены основные идеи, на которых базируется КОРСТ как макротеория. В ней совместно и равноправно учитываются стохастические воздействия квантового и теплового типов во всем диапазоне допустимых температур. В итоге основные макропараметры данной теории выражаются через константу Вина $\varkappa=\hbar / 2 k_{\mathrm{B}}$. Подтверждением справедливости КОРСТ в области достаточно низких температур могло бы служить обнаружение на опыте минимального, отличного от нуля отношения $\mathcal{J}^{*} / S^{*} \rightarrow \varkappa \neq 0$.

Первые указания на существенную роль величины $\varkappa$ были, по-видимому, получены в экспериментах Андроникашвили [29] по исследованию вязкости жидкого гелия ниже $\lambda$-точки. Постановка новых экспериментов в этой области (см. в этой связи [30]), а также анализ данных прежних экспериментов по изучению явлений переноса ниже температуры вырождения могли бы внести значительную ясность в эту проблему.

Другой областью физики, где могла бы проявиться фундаментальная роль константы $\varkappa$, является теория сильной связи в квантовой теории поля при конечных температурах, включая проблему конфайнмента и анализ свойств кварк-глюонной плазмы. Некоторые предвестники такой роли константы $\varkappa$ были обнаружены недавно в ходе опытов на ускорителе RHIC в Брукхейвене [31].

Основная проблема, которую предстоит решить при применении КОРСТ, - предложить в каждом конкретном случае критерий выбора характерной частоты объекта $\omega$, определяющей его минимальную эффективную температуру $T_{\min }^{*}$. По нашему мнению, наряду со стандартным сопоставлением такой частоты с энергией основного состояния объекта возможны и другие варианты подобного выбора. В частности, величину $T_{\text {min }}^{*}$, по-видимому, можно определять по характерным значениям частот, которые принято вводить в эффектах Казимира, Унру, Хокинга, при лазерном охлаждении атомов за счет эффекта Доплера, в квантовой хромодинамике в области конфайнмента или в современных версиях $\mathrm{AdS} / \mathrm{CFT-соответствия} \mathrm{в} \mathrm{кван-}$ товой теории поля при конечных температурах [31] и т.п. Более того, одному и 
тому же макрообъекту в термостате могут быть сопоставлены несколько независимых частот $\omega_{i}$, которым будут отвечать разные $\left(T_{\min }^{*}\right)_{i}($ например, температуры “замораживания" степеней свободы в двухатомных газах или температуры возникновения конденсатов). При этом во всех этих случаях критическую роль должна играть константа Вина $\varkappa$, характеризующая целостное стохастическое воздействие.

Разумеется, распространение понятий КОРСТ на объекты с непериодическими движениями и переход к анализу неравновесных процессов вблизи теплового равновесия потребуют дальнейшего развития изложенных здесь идей. Предполагается, что исследование затронутых здесь вопросов продолжится.

Благодарности. Автор выражает глубокую благодарность редколлегии журнала за предоставленную возможность опубликовать статью в номере, посвященном памяти Д.Н. Зубарева. Я имел честь общаться с Дмитрием Николаевичем на протяжении тридцати пяти лет и должен подчеркнуть, что он сыграл значительную роль в формировании моей научной и общественной позиции. Проявившийся у меня интерес к участию в квантовом обобщении равновесной статистической термодинамики, безусловно, отражает плодотворное влияние Дмитрия Николаевича, которое продолжает действовать до сих пор.

Особую благодарность я выражаю моим друзьям и коллегам, с которыми я имел возможность обсуждать пути обобщения термодинамики на протяжении последних десяти лет. Первым среди них, бесспорно, следует назвать А. Г. Башкирова, которого мы недавно потеряли. Я благодарю Н. М. Плакиду, В. И. Санюка, А. И. Оксака, А. Д. Гладуна, Ю.Г. Рудого за помощь в работе и нелицеприятную критику. Выражаю также благодарность В.А. Осипову и Ю.П. Рыбакову за стимулирующие литературные указания, В.Г. Барьяхтару, Э.А. Пашицкому и С. М. Рябченко за плодотворное обсуждение результатов, а также всем участникам семинаров ЛТФ ОИЯИ, РУДН, МФТИ, НИИЯФ МГУ, МИ РАН, БИТФ и Института магнетизма НАНУ за полезную дискуссию. Не могу не отметить неоценимую роль, которую сыграла на всех этапах данного исследования О. Н. Голубева.

Работа выполнена при поддержке РФФИ (грант № 07-06-00239).

\section{Список литературы}

[1] Дж. Гиббс, Основные принципы статистической механики, излагаемые со специальным применением к рациональному обоснованию термодинамики, Наука, М., 1982, гл. IX.

[2] A. Einstein, Ann. Phys., 14 (1904), 351; рус. пер.: А. Эйнштейн, "К общей молекулярной теории теплоты", Собрание научных трудов. Т. 3. Работы по кинетической теории, теории излучения и основам квантовой механики (1901-1955), Наука, М., 1966, 67.

[3] A. Einstein, Conseil de Physique, Inst. Solvay, 1911 Rapports, Gauthier-Villars, Paris, 1912, 407; pус. пер.: А. Эйнштейн, "К современному состоянию проблемы удельной теплоемкости", Собрание научных трудов. Т. 3. Работы по кинетической теории, теории излучения и основам квантовой механики (1901-1955), Наука, М., 1966, 277.

[4] А. Д. Суханов, ЭЧАЯ, 36:6 (2005), 1281.

[5] А. Д. Суханов, Ю. Г. Рудой, УФН, 176:5 (2006), 551.

[6] L. Gunther, J. Low Temper. Phys., 77:1-2 (1989), 151.

[7] J. Wu, A. Widom, Phys. Rev. E, 57:5 (1998), 5178.

[8] Д. Н. Зубарев, Неравновесная статистическая термодинамика, Физматлит, М., 1971.

[9] Л. Д. Ландау, Е. М. Лифшиц, Статистическая физика, Ч. 1, Физматлит, М., 2001. 
[10] Н. Н. Боголюбов, Лекции по квантовой статистике, Радянська школа, Киев, 1949; Собрание научных трудов. Т. 6. Равновесная статистическая механика, 1945-1986, Наука, М., 2006.

[11] Р. Фейнман, Статистическая механика. Курс лекиий, Мир, М., 1978.

[12] Д. Н. Зубарев, М. В. Токарчук, ТМФ, 88:2 (1991), 286.

[13] A. D. Sukhanov, "On the global interrelation between quantum dynamics and thermodynamics", Proc. XI Int. Conf. "Problems of Quantum Field Theory" (Dubna, 1998), JINR, Dubna, 1999, 232.

[14] A. Einstein, Ver. Deutsch. Phys. Ges., 16 (1914), 820; рус. пер.: А. Эйнштейн, "К квантовой теории", Собрание научных трудов. Т. 3. Работы по кинетической теории, теории излучения и основам квантовой механики (1901-1955), Наука, М., 1966, 328.

[15] F. Bloch, Z. Phys. A, 74 (1932), 295.

[16] E. Wigner, Phys. Rev., 40 (1932), 749.

[17] D. Han, Y. Kim, M. Noz, Coupled harmonic oscillators and Feynman's rest of the Universe, arXiv: cond-mat/9705029.

[18] Ю. Л. Климонтович, Статистическая теория открытых систем. Т. 3. Физика квантовых открытых систем, Янус, М., 2001.

[19] Yu. G. Rudoy, A. D. Sukhanov, Cond. Mat. Phys., 8:3(43) (2005), 507.

[20] L. Boltzmann, Vorlesungen uber die Prinzipien der Mechanik. Bd. II. Teil enthaltend: Die Wirkungsprinzipe, die Lagrangeschen Gleichungen und deren Anwendungen, J. A. Barth, Leipzig, 1904; рус. пер.: Л. Больцман, "Отрывок из “Лекций о принципах механики", Вариационные принципь механики, Физматлит, М., 1959, 468.

[21] С. А. Богуславский, "Основы молекулярной физики и применение статистики к вычислению термодинамических потенциалов. Глава III. Принцип наименьшего действия в термодинамике", Научные известия, сб. 3, Физика, Труды Московского физического общества им. П.Н. Лебедева, 1922, 17; Избраннъе труды по физике, Физматгиз, М., $1961,162$.

[22] L. de Broglie, La thermodynamique de la particule isolée, Gauthier-Villars, Paris, 1964.

[23] Х. Умэдзава, Х. Мацумото, М. Татики, Термополевая динамика и конденсированные состояния, Мир, М., 1985.

[24] H. Umezawa, Advanced Field Theory: Micro, Macro and Thermal Physics, AIP, New York, 1993.

[25] А. Д. Суханов, ЭЧАЯ, 36:7А (2005), 138.

[26] А. Д. Суханов, ТМФ, 148:2 (2006), 295.

[27] V. V. Dodonov, J. Opt. B, 4 (2002), S98.

[28] В. В. Додонов, В. И. Манько, Тр. ФИАН, 183 (1987), 5.

[29] Э. Л. Андроникашвили, ЖЭТФ, 18:5 (1948), 424.

[30] Э. А. Пашицкий, С. М. Рябченко, ФНT, 33:1 (2007), 12.

[31] D. Son, A. Starinets, Ann. Rev. Nucl. Part. Sci., 57 (2007), 95; arXiv: hep-th/0704.0240.

Поступила в редакцию 13.06.2007, после доработки 1.10.2007 